\title{
Specification, Verification, and Synthesis of Concurrency Control Components *
}

\author{
Tuba Yavuz-Kahveci Tevfik Bultan \\ Computer Science Department \\ University of California \\ Santa Barbara, CA 93106, USA \\ \{tuba,bultan\}@cs.ucsb.edu
}

\begin{abstract}
Run-time errors in concurrent programs are generally due to the wrong usage of synchronization primitives such as monitors. Conventional validation techniques such as testing become ineffective for concurrent programs since the state space increases exponentially with the number of concurrent processes. In this paper, we propose an approach in which 1) the concurrency control component of a concurrent program is formally specified, 2) it is verified automatically using model checking, and 3) the code for concurrency control component is automatically generated. We use monitors as the synchronization primitive to control access to a shared resource by multiple concurrent processes. Since our approach decouples the concurrency control component from the rest of the implementation it is scalable. We demonstrate the usefulness of our approach by applying it to a case study on Airport Ground Traffic Control.

We use the Action Language to specify the concurrency control component of a system. Action Language is a specification language for reactive software systems. It is supported by an infinite-state model checker that can verify systems with boolean, enumerated and unbounded integer variables. Our code generation tool automatically translates the verified Action Language specification into a Java monitor. Our translation algorithm employs symbolic manipulation techniques and the specific notification pattern to generate an optimized monitor class by eliminating the context switch overhead introduced as a result of unnecessary thread notification. Using counting abstraction, we show that we can automatically verify the monitor specifications for arbitrary number of threads.
\end{abstract}

\section{Keywords}

concurrent programming, monitors, infinite-state model

\footnotetext{
${ }^{*}$ This work is supported in part by NSF grant CCR-9970976 and NSF CAREER award CCR-9984822.
}

Permission to make digital or hard copies of all or part of this work for personal or classroom use is granted without fee provided that copies are not made or distributed for profit or commercial advantage and that copies bear this notice and the full citation on the first page. To copy otherwise, to republish, to post on servers or to redistribute to lists, requires prior specific permission and/or a fee.

Copyright 2001 ACM X-XXXXX-XX-X/XX/XX ...\$5.00. checking, specification languages

\section{INTRODUCTION}

Writing a concurrent program is an error prone task. A concurrent programmer has to keep track of not only the possible values of the variables of the program, but also the states of its concurrent processes. Failing to use concurrency constructs such as semaphores or monitors correctly results in run-time errors such as deadlocks and violation of safety properties. Conventional validation techniques such as testing become ineffective for concurrent programs since the state space of a concurrent program increases exponentially both with the number of variables and the number of concurrent processes in it.

Monitors are a programming language construct introduced to ease the difficult task of concurrent programming [14]. Structured programming languages help programmers in keeping track of the states of the program variables by providing abstractions such as procedures and associated scoping rules to localize variable access. Monitors are a similar mechanism for structuring concurrent programs, they provide scoping rules for concurrency.

Since monitors are an integral part of Java, recently, concurrent programming using monitors gained increased attention [16]. A monitor consists of a set of variables shared among multiple processes and a set of associated procedures for accessing them. Shared variables of a monitor are not accessible outside of its procedures. At any time, only one process is allowed to be active in a monitor. Processes synchronize using specific operations which lets them wait (i.e., sleep) until they receive a signal from another process. Wait and signal operations are coordinated using condition variables. Even though monitors provide a better abstraction for concurrent programming compared to other constructs such as semaphores, they are still error prone. Coordinating wait and signal operations on several condition variables among multiple processes can be very challenging even for the implementation of simple algorithms.

In this paper we propose a new approach for developing reliable concurrent programs. First aspect of our approach is to start with a specification of the concurrency control component of the program rather than its implementation. We use monitors as the underlying concurrency control primitive. We present a monitor model in Action Language [4]. Action Language is a specification language for reactive software systems. It supports both synchronous and asynchronous compositions and hierarchical specifications. We 
show that monitors can be specified in Action Language using asynchronously composed modules. Our monitor model in Action Language has one important aspect, it does not rely on condition variables. Semantics of Action Language lets us get rid of condition variables (with associated wait and signal operations) which simplifies the specification of a monitor significantly.

Most important component of our approach is the use of an automated verification tool for checking properties of monitor specifications. Action Language is supported by an infinite-state model checker that can verify or falsify (by producing counter-example behaviors) both invariance and liveness (or any CTL) properties of Action Language specifications [6]. In this paper we focus on verification of monitor invariants, however, the approach presented in this paper can be extended to universal portion of the temporal logic CTL. For the infinite-state systems that can be specified in Action Language, model checking is undecidable. Hence our verifier uses various heuristics to guarantee convergence. It does not produce false positives or false negatives but its analysis can be inconclusive.

The last component of our approach is a code generation algorithm for synthesizing monitors from Action Language specifications. Our goal is not generating complete programs, rather, we are proposing a modular approach for generating concurrency control component of a program that manipulates shared resources.

We use a case study on Airport Ground Traffic Control [21] to show the effectiveness and scalability of our technique. This case study uses a fairly complex airport ground network similar to that of Seattle Tacoma International Airport. Our model checker can verify all the safety properties of the specification for this case study and our code generation tool automatically generates an optimized Java class (in terms of the context switch overhead that would be incurred in a multithreaded application).

Recently, there has been several attempts in adopting model checking to verification of concurrent programs [8, 13]. These approaches translate a concurrent Java program to a finite model and then check it using available model checking tools. Hence, they rely on the ability of model checkers to cope with the state space explosion problem. However, to date, model checkers are not powerful enough to check implementations of concurrent programs. Hence, most of the recent work on verification of concurrent programs have been on efficient model construction from concurrent programs $[8,13,11]$.

Our approach provides a different direction for creating reliable concurrent programs. It has several advantages: 1) It avoids the implementation details in the program which do not relate to the property to be verified. 2) There is no model construction problem since the specification language used has a model checker associated with it. 3) By pushing the verification to an earlier stage in software development (to specification phase rather than the implementation phase) it reduces the cost of fixing bugs. However, our approach is unlikely to scale to generation of complete programs. This would require the specification language to be more expressive and would introduce a model construction problem at the specification stage. Hence we focus on synthesizing concurrency control components which are correct by construction and can be integrated to a concurrent program safely. Another aspect of our approach which is different from the previous work is the fact that we are using an infinite state model checker rather then finite state techniques. Using our infinite state model checker we can verify properties of specifications with unbounded integer variables and arbitrary number of threads.

While this work was under review, independently, Deng et al. also presented an approach that combines specification, synthesis and verification for concurrent programs [12]. One crucial difference between our approach and the approach presented in [12] is apparent in the (otherwise remarkably similar) titles. In our approach automated verification is performed on the specification, not on the implementation. Hence, our approach shields the automated verification tool from the implementation details.

Rest of the paper is organized as follows. In Section 2 we describe our case study. In Section 3 we explain concurrency control with monitors and their implementation in Java. In Section 4 using our case study we discuss how monitors can be specified in Action Language. We also present a general template for specifying monitors in Action Language in that section. In Section 5 we discuss how monitor specifications can be automatically verified using Action Language Verifier. We also show that using counting abstraction monitor specifications can be verified for arbitrary number of threads. In Section 6 we present the algorithms for automatically generating Java monitor classes from monitor specifications. Finally, in Section 7 , we state our conclusions and directions for future work.

\section{AN AIRPORT GROUND TRAFFIC CONTROL CASE STUDY}

We will present an Airport Ground Traffic Control case study to demonstrate the techniques proposed in this paper. Airport Ground Traffic Control handles allocation of airport ground network resources such as runways, taxiways, and gates to the arriving and departing airplanes. Airport Ground Traffic Control is safety critical. $51.5 \%$ of hull loss accidents from 1959 through 1996 were associated with airport ground operations such as taxi, takeoff, and landing [1]. Simulations play an important role for airport safety since they enable early prediction of possible runway incursions which is a growing problem at busy airports throughout the world. [21] presents a concurrent simulation program for modeling Airport Ground Traffic Control using Java threads. In this paper, we demonstrate that concurrency control component of such a program can be formally specified, automatically verified, and synthesized in our framework. We use the same airport ground network model used in [21] (shown in Figure 1) similar to Seattle/Tacoma International Airport. There are two runways: $16 \mathrm{R}$ and $16 \mathrm{~L}$. Runway $16 \mathrm{R}$ is used by arriving airplanes during landing. After landing, an arriving airplane takes one of the exits C3-C8. After taxiing on C3-C8, the arriving airplanes need to cross runway $16 \mathrm{~L}$. After crossing $16 \mathrm{~L}$, they continue on one of the taxiways B2, B7, B9-B11 and reach the gates in which they park. Departing airplanes use runway $16 \mathrm{~L}$ for takeoff. The control logic for ground traffic of this airport must implement the following rules:

1. An airplane can land (takeoff) using $16 \mathrm{R}(16 \mathrm{~L})$ only if no airplane is using $16 \mathrm{R}(16 \mathrm{~L})$ at the moment.

2. An airplane taxiing on one of the exits C3-C8 can cross 


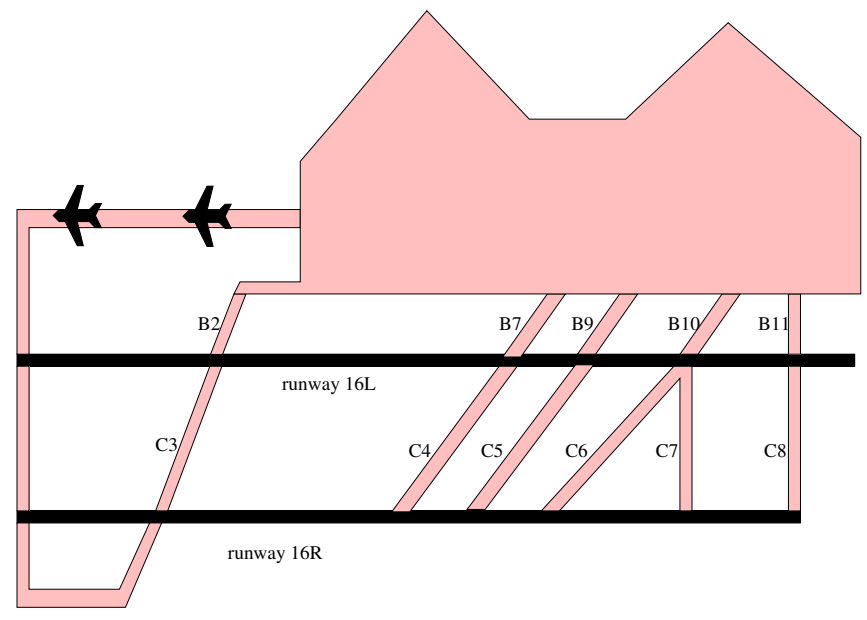

Figure 1: An airport ground network similar to that of the Seattle Tacoma International Airport

runway $16 \mathrm{~L}$ only if no airplane is taking off at the moment.

3. An airplane can start using $16 \mathrm{~L}$ for taking off only if none of the crossing exits C3-C8 is occupied at the moment. (Arriving airplanes have priority over departing airplanes.)

4. Only one airplane can use a taxiway at a time.

We give the Action Language specification of the Airport Ground Traffic Control system in Section 4. In Section 5 we discuss how we used the Action Language Verifier to automatically verify the properties of this system. In Section 6 we show the Java monitor class synthesized from the Action Language specification.

\section{CONCURRENCY CONTROL WITH MONITORS}

A monitor is a synchronization primitive that is used to control access to a shared resource by multiple concurrent processes. A monitor consists of a set of variables and procedures with the following rules: 1) The variables in a monitor can only be accessed through the procedures of the monitor. 2) No two processes can execute procedures of the monitor at the same time. We can view the second rule as monitor having a mutual exclusion lock. Only the process that has the monitor lock can be active in the monitor. Any process that calls a monitor procedure has to acquire the monitor lock before executing the procedure and release it after it exits. This synchronization is provided implicitly by the monitor semantics, hence, the programmer does not have to explicitly write the acquire lock and release lock operations.

Monitors provide additional synchronization operations among processes based on condition variables. Two operations on condition variables are defined: wait and signal. A process that performs a wait operation on a condition variable sleeps and releases the monitor lock. It can only be awakened by a signal operation on the same condition variable. A waiting process that has been awakened has to re-acquire the monitor lock before it resumes operation. If there are no waiting processes, then signal operation is ignored (and forgotten, i.e., it does not affect processes which execute a wait later on). Wait and signal operations can be implemented using one wait queue per condition variable. When a process executes the wait operation on a condition variable it enters the corresponding wait queue. A signal operation on a condition variable removes one process from the corresponding wait queue and resumes its operation (after re-acquiring the monitor lock). In signal and continue semantics for the signal operation, signaling process keeps the monitor lock until it exits or waits. Different semantics and additional operations have also been used for signaling such as signal and wait semantics and signalAll operation [3].

Typically condition variables are used to execute a set of statements only after a guarding condition becomes true. To achieve this, a condition variable is created that corresponds to the guarding condition. The process which will execute the guarded statements tests the guarding condition and calls the wait on the corresponding condition variable if the guarding condition is false. Each process which executes a statement that can change the truth value of the guarding condition signals this to the processes that are waiting on the corresponding condition variable.

State of a monitor is represented by its variables. Set of states that are safe for a monitor can be expressed as a monitor invariant [3]. Monitor invariant is expected to hold when no process is accessing the monitor (i.e., it is not guaranteed to hold when a process is active within a monitor procedure).

\subsection{Monitors in Java}

Java is an object-oriented programming language that supports concurrent programming via threads and monitors. Each Java object has a mutual exclusion lock. A monitor in Java is implemented using the object locks and the synchronized keyword. A block of statements can be declared to be synchronized using the lock of an object o as synchronized (o) $\{\ldots\}$. This block can only be executed after the lock for the object o is acquired. Methods can also be declared to be synchronized which is equivalent to enclosing the method within a synchronized block using object this, i.e., synchronized(this) $\{\ldots\}$. A monitor object in Java is created by declaring a class with private variables that correspond to shared variables of the monitor. Then each monitor procedure is declared as a synchronized method to meet the mutual exclusion requirement.

Wait and signal operations are implemented as wait and notify methods in Java. However, in Java, each object has only one wait queue. This means that when there is a notify call, any waiting process in the monitor can wake up. If there is more than one condition that processes can be waiting for, awakened processes have to recheck the conditions they have been waiting for. Note that, if a process that was waiting for a different condition is awakened, then the notify call is lost. This can be prevented by using notifyAll method which wakes up all the waiting processes.

Using a single wait queue and notifyAll method one can safely implement monitors in Java. However, such an implementation will not be very efficient. To get better efficiency, one can use other objects (declared as members of the monitor class) as condition variables together with synchronized blocks on those objects. Since each object has a lock and 
an associated wait queue, this makes it possible to put processes waiting on different conditions to different queues. However, this implies that there will be more than one lock used in the monitor. (In addition to monitor lock there will be one lock per condition variable.) Use of multiple locks in Java monitor classes is prone to deadlocks and errors [16].

\section{SPECIFICATION OF MONITORS}

Although monitors provide a higher level of abstraction for concurrent programs compared to mechanisms such as semaphores, they can still be tedious and difficult to implement. We argue that Action Language can be used to specify monitors in a higher level of abstraction. Monitor specifications in Action Language do not rely on condition variables. Since in Action Language an action is executed only when its guard evaluates to true, we do not need conditional waits.

Figure 2 shows the Action Language specification of the Airport Ground Traffic Control case study. An Action Language specification consists of a set of module definitions. A module definition consists of variable declarations, a restrict expression, an initial expression, submodule definitions, action definitions, and a module expression. Semantically, each module corresponds to a transition system with a set of states, a set of initial states and a transition relation. Variable declarations define the set of states of the module.

In Figure 2 we implemented the shared resources of the Airport Ground Traffic Control, which are runways and taxiways, as integer variables. Variables numRW16R and numC3 denote the number of airplanes on runway $16 \mathrm{R}$ and on taxiway $\mathrm{C} 3$, respectively. We use enumerated variables (local variable pc in submodule Airplane) to encode states of an airplane. An arriving airplane can be in one of the following states: arFlow, touchDown, taxiToXY, taxiFrXY and parked, where the state arFlow denotes that the airplane is in the air approaching to the airport, the state touchDown denotes that the airplane has just landed and is on the runway $16 \mathrm{R}$, the state taxiToXY denotes that the airplane is currently in the taxiway $\mathrm{Y}$ and is going to cross the runway $\mathrm{X}$, the state taxiFrXY denotes that the airplane is currently in the taxiway $\mathrm{Y}$ and has just crossed the runway $\mathrm{X}$, and finally, the state parked denotes that the airplane is parked at the gate. Similarly, a departing airplane can be in one of the following states: parked, take0ff, and depFlow, where the state parked denotes that the airplane is parked at the gate, the state take0ff denotes that the airplane is taking off from the runway $16 \mathrm{~L}$, and the state depFlow denotes that the airplane is in the air departing from the airport.

Action Language is a modular language. An Action Language specification can be defined in terms of a hierarchy of modules. In Figure 2 module main has a submodule Airplane. Submodule Airplane models both arriving and departing airplanes and corresponds to a process type (or thread class in Java). Submodule Airplane has one local boolean variable (pc) which is used to keep track of the states an airplane can be in. Note that, each instantiation of a module will create different instantiations of its local variables.

Set of states can be restricted using a restrict expression. In Figure 2, variables numC3, numRW16R, and numRW16L are restricted to be greater than or equal to 0 . Initial expression defines the set of initial states of a module. For instance, in Figure 2 variables numRW16R, numRW16L, and numC3 are ini- tialized 0. Initial and restrict expressions of the submodules are conjoined with the initial and restrict expressions of the main module to obtain the overall initial condition and the restrict expression, respectively.

Behavior of a module (i.e., its transition relation) is defined using a module expression. A module expression (which starts with the name of the module) is written by combining its actions and submodules using asynchronous (denoted by I) and/or synchronous (denoted by \&) composition operators. For instance, module Airplane is defined in terms of asynchronous composition of its actions reqLand, exitRW3, and so on. Module main (which defines the transition relation of the overall system) is defined in terms of asynchronous composition of multiple instantiations of its submodule Airplane. The specification in Figure 2 specifies a system with more than two airplane processes. In Figure 2 only asynchronous composition is used.

Each atomic action in Action Language defines a single execution step. In an action expression for an action $a$, primed (or range) variables, $\operatorname{RVAR}(a)$, denote the next-state values for the variables and unprimed (or domain) variables, $\operatorname{DVAR}(a)$, denote the current-state values. For instance, action exitRW3 in module Airplane indicates that when an arriving airplane is in the touch-down state ( $\mathrm{pc}=$ touchDown) if taxiway $\mathrm{C} 3$ is available (numC3 $=0$ ) then in the next state runway $16 R$ will have one less airplane (numRW16R' =numRW16R-1) and taxiway C3 will be used by one more airplane (numC3'=numC3+1) and the airplane will be in state $\mathrm{pc}^{\prime}=\mathrm{taxiTo16LC3}$. Note that an airplane taxiing on taxiway $\mathrm{C} 3$ crosses runway $16 \mathrm{~L}$ on its route and continues on taxiway B2 (see Figure 1).

Asynchronous composition of two actions is defined as the disjunction of their transition relations. However, we also assume that an action preserves the values of the variables which are not modified by itself. Formally, we extend the action expression $\operatorname{EXP}\left(a_{1}\right)$, for action $a_{1}$, by conjoining it with a frame constraint $\operatorname{EXP}^{\prime}\left(a_{1}\right) \equiv \operatorname{EXP}\left(a_{1}\right) \wedge \operatorname{SAME}\left(\operatorname{RVAR}\left(a_{2}\right)-\right.$ $\left.\operatorname{RVAR}\left(a_{1}\right)\right)$ where $\operatorname{Same}(V)$ denotes $\wedge v_{i} \in V v_{i}^{\prime}=v_{i}$ and denotes set difference. Similarly, we extend the expression for $a_{2}, \operatorname{EXP}\left(a_{2}\right)$, to $\operatorname{EXP}^{\prime}\left(a_{2}\right)$. Then, we define $\operatorname{EXP}\left(a_{1} \mid a_{2}\right)$ as

$$
\operatorname{EXP}\left(a_{1} \mid a_{2}\right) \equiv \operatorname{EXP}^{\prime}\left(a_{1}\right) \vee \operatorname{EXP}^{\prime}\left(a_{2}\right)
$$

Asynchronous composition of two modules is defined similarly.

In Figure 2 monitor invariants that we expect the system to satisfy is written using the spec, invariant and next keywords at the end of the main module. In Action Language keywords invariant, eventually and next are synonyms for CTL operators AG, AF, and AX, respectively.

The specification given in Figure 2 specifies a solution to the Airport Ground Traffic Control without specifying the details about the implementation of the monitor. It is a high level specification compared to a monitor implementation, in the sense that, it does not introduce condition variables and waiting and signaling operations which are error prone.

We give a general template for specifying monitors in Action Language in Figure 3. It consists of a main module $m$ and a list of submodules $m_{1}, \ldots, m_{n}$. The variables of the main module (denoted $\operatorname{var}(m)$ ) define the shared variables of the monitor specification. Currently, available variable types in Action Language are boolean, enumerated and integer. This restriction comes from the symbolic manipulation 


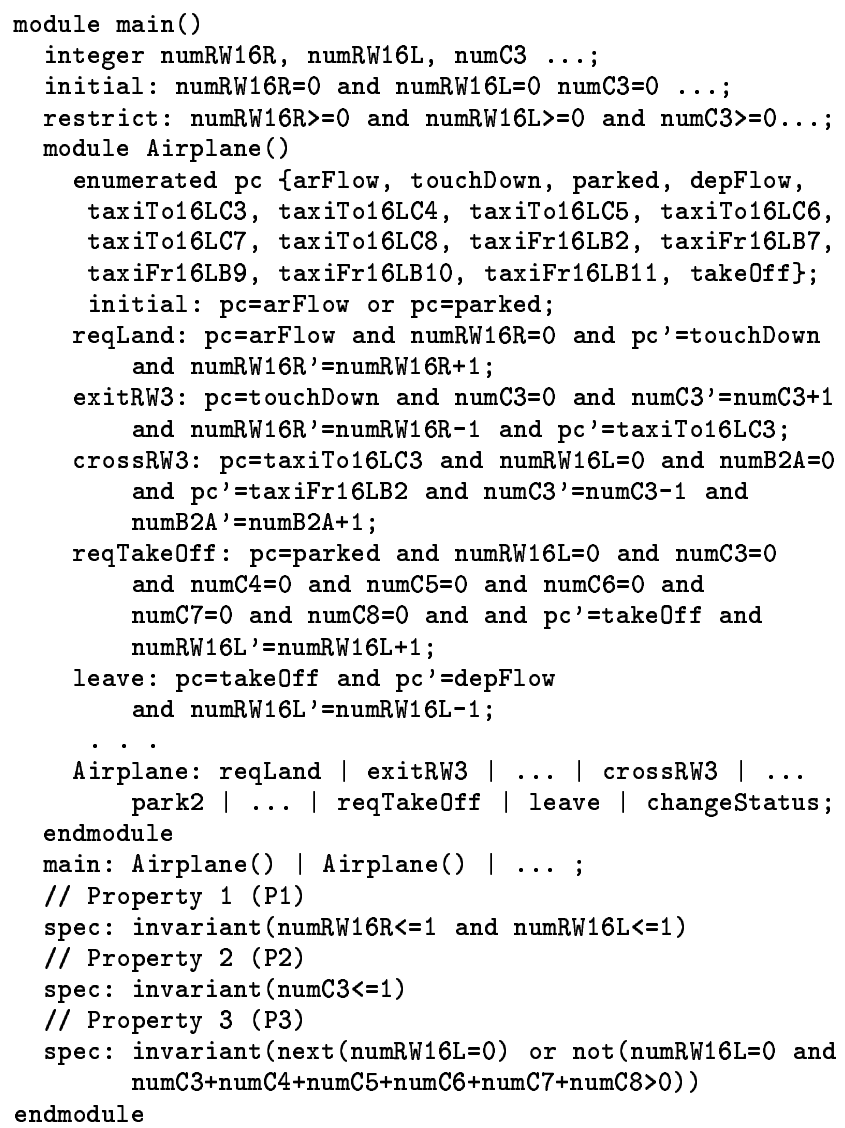

Figure 2: Action Language Specification of the Airport Ground Traffic Control Case Study

capabilities of the Action Language Verifier (which can be extended as we discuss in [15]). We also allow declaration of parameterized constants. For example, a declaration such as parameterized integer size would mean that size is an unspecified integer constant, i.e., when a specification with such a constant is verified it is verified for all possible values of size.

Each submodule $m_{i}$ corresponds to a process type, i.e., each instantiation of a submodule corresponds to a process. Each submodule $m_{i}$ has a set of local variables $\left(\operatorname{VAR}\left(m_{i}\right)\right)$ and atomic actions $\left(\mathrm{ACT}\left(m_{i}\right)\right)$. Note that, in a monitor specification our goal is to model only the behavior of a process that is relevant to the properties of the monitor. Therefore, local variables $\operatorname{VAR}\left(m_{i}\right)$ of a submodule should only include the variables that are relevant to the correctness of the monitor. The transition relation of a submodule is defined as the asynchronous execution of its atomic actions.

To simplify the abstraction and code generation algorithms we will present in the following sections we restrict the form of action expressions as follows: Given an action $a \in \operatorname{ACT}\left(m_{i}\right), \operatorname{EXP}(a)$ can be written as

$$
\operatorname{EXP}(a) \equiv d_{l}(a) \wedge r_{l}(a) \wedge d_{s}(a) \wedge r_{s}(a)
$$

where $d_{l}(a)$ is an expression on unprimed local variables of module $m_{i}\left(\operatorname{VAR}\left(m_{i}\right)\right), r_{l}(a)$ is an expression on primed and unprimed local variables of $m_{i}, d_{s}(a)$ is an expression on unprimed shared variables $(\operatorname{VAR}(m))$, and $r_{s}(a)$ is an expression

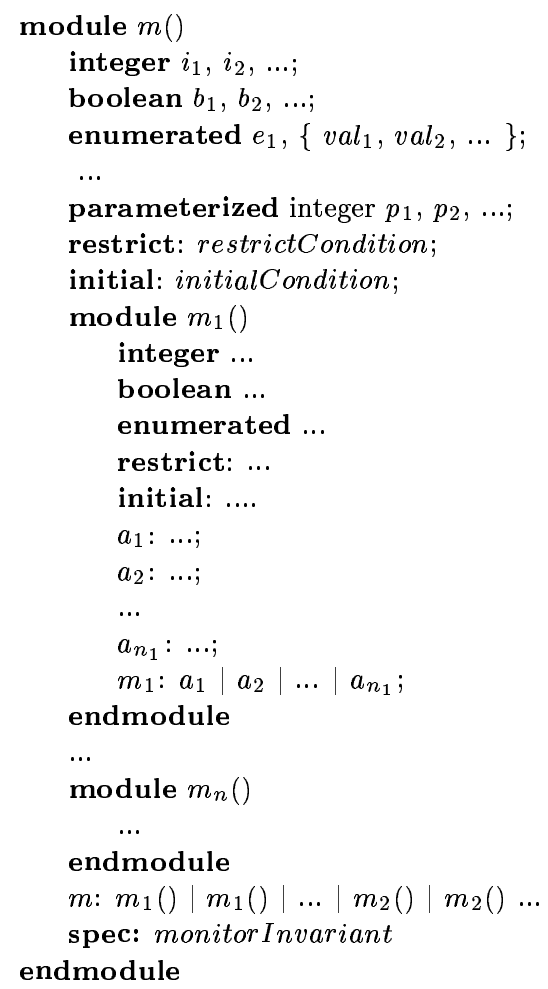

Figure 3: A Monitor Template in Action Language

on primed and unprimed shared variables. For example, for the action exitRW3 in Figure 2

$$
\begin{aligned}
& d_{l}(a) \equiv \mathrm{pc}=\text { touchDown } \\
& r_{l}(a) \equiv \mathrm{pc}=\text { taxiTo16LC3 } \\
& d_{s}(a) \equiv \text { numC3 }=0 \\
& r_{s}(a) \equiv \text { numRW16R' }=\text { numRW16R-1 and numC3' =numC3+1 }
\end{aligned}
$$

In the template given in Figure 3, transition relation of the main module $m$ is defined as the asynchronous composition of its submodules, which defines the behavior of the overall system.

\section{VERIFICATION OF MONITOR SPECIFICATIONS}

Action Language Verifier [6] consists of 1) a compiler that converts Action Language specifications to composite symbolic representations, and 2) an infinite-state symbolic model checker which verifies (or falsifies) CTL properties of Action Language specifications. Action Language compiler translates an Action Language specification to a transition system $T=(S, I, R)$ that consists of a state space $S$, a set of initial states $I \subseteq S$, and a transition relation $R \subseteq S \times S$. Unlike the common practices in model checking, $S$ can be infinite and $R$ may not be total (i.e., there maybe states $s \in S$ for which there does not exist a $s^{\prime}$ such that $\left(s, s^{\prime}\right) \in R$ ). For the infinite state systems that can be specified in Action Language, model checking is undecidable. Action Language Verifier uses several heuristics to achieve convergence such as approximations based on truncated fixpoint computations and widening, loop-closures and approximate reachability analysis. Since we allow non-total transition systems also some fixpoint computations have to be modified [6]. 
For the monitor model given in Figure 3, the state space $S$ is obtained by taking the Cartesian product of the domains of the shared variables of the main module $(\operatorname{VAR}(m))$ and the domains of the local variables of each submodule $\left(\operatorname{VAR}\left(m_{i}\right)\right)$ for each instantiation. The transition relation of $R$ of the overall system is defined as

$$
R \equiv \bigvee_{i, j, k} r_{i j k}
$$

where $r_{i j k}$ corresponds to the action expression of action $a_{k}$ in instantiation $j$ of module $m_{i}$. Action Language parser renames local variables of each submodule $m_{i}$ for each instantiation $j$ to obtain $r_{i j k}$ 's. Also, as explained above, action expressions are automatically transformed by the Action Language parser by adding the frame constraints (unmodified variables preserve their value). Initial states of the system is defined as

$$
I \equiv I_{m} \wedge \bigwedge_{i, j} I_{i j}
$$

where $I_{m}$ denotes the initial condition for main module $m$, and $I_{i j}$ denotes the initial condition for instantiation $j$ of module $m_{i}$.

Composite Symbolic Library [15] is the symbolic manipulator used by the Action Language Verifier. It combines different symbolic representations using the composite model checking approach [5]. Generally, model checking tools have been built using a single symbolic representation such as BDDs [17] or polyhedra [2]. A composite model checker combines different symbolic representations to improve both the efficiency and the expressiveness of model checking. Our current implementation of the Composite Symbolic Library uses two basic symbolic representations: BDDs (for boolean and enumerated variables) and polyhedral representation (for integers). Since Composite Symbolic Library uses an object-oriented design, Action Language Verifier is polymorphic. It can dynamically select symbolic representations provided by the Composite Symbolic Library based on the variable types in the input specification. For example, if there are no integer variables in the input specification Action Language Verifier becomes a BDD-based model checker.

To analyze a system using Composite Symbolic Library, one has to specify its initial condition, transition relation, and state space using a set of composite formulas. A composite formula is obtained by combining integer arithmetic formulas on integer variables with boolean variables using logical connectives. Enumerated variables are mapped to boolean variables by the Action Language parser. Since integer representation in the Composite Symbolic Library currently supports only Presburger arithmetic, we restrict arithmetic operators to + and - . However, we allow multiplication with a constant and quantification.

A composite formula, $p$, is represented in disjunctive normal form as

$$
p \equiv \bigvee_{i=1}^{n} \bigwedge_{t=1}^{T} p_{i t}
$$

where $p_{i t}$ denotes the formula of basic symbolic representation type $t$ in the $i$ th disjunct, and $n$ and $T$ denote the number of disjuncts and the number of basic symbolic representation types, respectively. Our Composite Symbolic Library implements methods such as intersection, union, complement, satisfiability check, subset test, which manipulate composite representations in the above form. These methods in turn call the related methods of basic symbolic representations.

Action Language Verifier iteratively computes the fixpoints for the temporal operators using the symbolic operations provided by the Composite Symbolic Library. Action Language Verifier uses techniques such as truncated fixpoints, widening and collapsing operators to compute approximations of the divergent fixpoint computations [6]. However, Action Language Verifier does not generate false negatives or false positives. It either verifies a property or generates a counter-example or reports that the analysis is inconclusive. This is achieved by using appropriate type of approximations for the fixpoints (lower or upper approximation) based on the temporal property and the type of the input query (which could be verify or falsify).

\subsection{Counting Abstraction}

In the Action Language template for monitor specifications (Figure 3), each submodule is instantiated a fixed number of times. This means that the specified system has a fixed number of processes. For example, the specification in Figure 2 describes a system with a specific number of airplane processes and hence, any verification result obtained for this specification is only guaranteed for a system with a specific number of airplane processes. In this section we will present the adaptation of an automated abstraction technique called counting abstraction [9] to verification of monitor specifications in Action Language. Using counting abstraction one can automatically verify the properties of a monitor model for arbitrary number of processes. The basic idea is to define an abstract transition system in which the local states of the processes are abstracted away but the number of processes in each local state is counted by introducing a new integer variable for each local state. For this abstraction technique to work we need local states of submodules to be finite. For example, if a submodule has a local variable that is an unbounded integer, we cannot use the counting abstraction. Note that, shared variables (i.e. $\operatorname{VAR}(m))$ can still be unbounded since they are not abstracted away.

Consider the specification in Figure 2. Each Airplane process has 16 local states. Note that, in the general case (Figure 3), each local state corresponds to a valuation of all the local variables of a submodule, i.e., the set of local states of a submodule is the Cartesian product of the domains of the local variables of that submodule. Given a module $m_{i}$, let $S_{i}$ be the set of all possible valuations of its local variables $\operatorname{VAR}\left(m_{i}\right)$, then we call $S_{i}$ the set of local states of $m_{i}$. In the counting abstraction, we introduce a nonnegative integer variable to represent each local state of each submodule. I.e., for each submodule $m_{i}$ and for each local state $s \in S_{i}$ of module $m_{i}$ we declare a nonnegative integer variable $i_{s}$. For example, for the specification in Figure 2, we introduce 16 integer variables for the Airplane submodule: arFlowC for state $\mathrm{pc}=\mathrm{arFlow}$ and depFlowC for state $\mathrm{pc}=\mathrm{depFlow}$. These variables will represent the number of processes that are in the local state that corresponds to them. For example, if arFlowC is 2 in the abstract system, this will imply that there are 2 processes in the corresponding states of the concrete system where pc=arFlow holds. Note that, there could be more than one concrete state that corresponds to an ab- 
stract state.

Once we defined the mapping between the states of the concrete system and the abstract system, next thing to do is to define the abstract transition relation, i.e., to translate the actions of the original system to the actions of the abstract system. Consider action exitRW3 in Figure 2. To translate this action to an action on the abstract system we only have to change the part of the expression using the current and next state local variables (i.e., pc=touchDown and pc'=taxiTo16L3). The part of the expression on current and next state shared variables (i.e., numC3 $=0$ and numRW16R' =numRW16R-1 and numC3'=numC3+1) will remain the same. Since we restricted all local variables to be finite, without loss of generality, we can assume that all local variables are boolean variables (as we discussed in Section 5 Action Language Verifier translates enumerated variables to boolean variables). As we stated in Section 4 we assume that the action expression is in the form:

$$
\operatorname{EXP}(a) \equiv d_{l}(a) \wedge r_{l}(a) \wedge d_{s}(a) \wedge r_{s}(a)
$$

where $d_{l}(a)$ is a boolean logic formula on local domain variables and $r_{l}(a)$ is a boolean logic formula on local domain variables. Since we are assuming that local variables can only be boolean, we do not need to have domain variables in $r_{l}(a)$. We can transform any action expression to a set of equivalent action expressions in this form by splitting disjuncts that involve both local and shared variables. For the action exitRW3 $d_{l}$ is pc=touchDown and $r_{l}$ is pc'=taxiTo16L3.

Let $S_{d} \subseteq S_{i}$ be the set of local states of the module $m_{i}$ which satisfy expression $d_{l}$. We translate $d_{l}$ to an expression on the variables of the abstract transition system by generating the expression

$$
d_{l}^{a} \equiv \sum_{s \in S_{d}} i_{s}>0
$$

where $i_{s}$ is the integer variable that represents the local state $s$. Note that, $d_{l}^{a}$ indicates that there exists a process which is in a local state that satisfies $d_{l}$. For exitRW3 the formula we obtain is simply touchDownC $>0$, i.e., there exists a process in the local state $\mathrm{pc}=$ touchDown.

Let $S_{r} \subseteq S_{i}$ be the set of local states of the module $i$ which satisfy expression $r_{l}$. We translate $r_{l}$ to an expression on the variables of the abstract transition system by generating the expression

$$
\begin{aligned}
r_{l}^{a} \equiv \bigvee_{s \in S_{d}, t \in S_{r}, s \neq t}\left(i_{t}^{\prime}=i_{t}+1 \wedge i_{s}^{\prime}=i_{s}-1\right. \\
\left.\wedge \bigwedge_{p \neq s, p \neq t} i_{p}^{\prime}=i_{p}\right) \\
\quad \vee \bigvee_{s \in\left(S_{d} \cap S_{r}\right)}\left(i_{s}^{\prime}=i_{s} \wedge \bigwedge_{p \neq s} i_{p}^{\prime}=i_{p}\right)
\end{aligned}
$$

The first disjunction enumerates all possible local current and next state pairs for the action and updates the counters accordingly. The second disjunct takes into account the cases where the local state of the process does not change. For the action exitRW3 we obtain the following expression: touchDownC'=touchDownC -1 and $\operatorname{taxiTo16L3C^{\prime }=}$ taxiTo16L3C'+1. Then, the abstraction of action exitRW3 is:

exitRW3: touchDownC $>0$ and numC3=0 and numC3' $=$ numC $3+1$ and numRW16R'=numRW16R-1 and touchDownC'=touchDownC-1 and $\operatorname{taxiTo16L3C'=taxiTo16L3C+1;~}$
After generating the abstract state-space and the abstract transition relation, last component of the abstraction is to translate the initial states. First, for each submodule $m_{i}$ in Figure 3 we declare a nonnegative parameterized integer constant $n u m_{-} m_{i}$ which denotes the number of instances of module $m_{i}$. By declaring this constant parameterized we guarantee that the verified properties will hold for all possible number of instantiations of each submodule. Let init $_{i}$ denote the local initial expression of a submodule $m_{i}$ and let $S_{\text {init }}$ denote the set of local states of the module $m_{i}$ which satisfy expression init $_{i}$. Then we add the following constraint to the initial expression of the abstract transition system:

$$
i n i t_{i}^{a} \equiv \sum_{s \in S_{i n i t}} i_{s}=n u m_{-} m_{i} \wedge \bigwedge_{s \notin S_{\text {in } i t}} i_{s}=0
$$

For the specification in Figure 2 we create a nonnegative parameterized integer constant numAirplane. Using the initial condition of the submodule Airplane, which is $\mathrm{pc}=\mathrm{arFlow}$ or $\mathrm{pc}=$ parked, we obtain the following constraint

arFlowC + parkedC=numAirplane and touchDownC $=0$ and $\operatorname{taxiTo16LC3C}=0$ and $\operatorname{taxiTo16LC4C}=0 \ldots$

and replace the initial constraint of Airplane submodule with this new constraint.

One can show that the monitor invariants verified on the abstract specification generated by the counting abstraction are also satisfied by the original monitor specification for arbitrary number of processes. This can be shown by defining an abstraction function between the state space of the original specification and the state space of the abstract specification generated by the counting abstraction [10].

for each submodule $m_{i}$ in $m$ do

Declare a parameterized integer num_ $m_{i}$ in $m$

Add num_ $m_{i} \geq 0$ to the restrict expression of $m$

Remove local variable declarations of module $m_{i}$

for each local state $s \in S_{i}$ of module $m_{i}$ do Declare an integer variable $i_{s}$ in $m_{i}$

Add $i_{s} \geq 0$ to the restrict expression of $m_{i}$

Replace initial expression init $_{i}$ of module $m_{i}$ with

$\sum_{s \in S_{\text {init }_{i}}} i_{s}=n u m \_m_{i} \wedge \bigwedge_{s \notin S_{\text {init }_{i}}} i_{s}=0$

where $S_{\text {init }_{i}}$ are the set of local states of $m_{i}$ which satisfy init $_{i}$

for each action $a$ in module $m_{i}$ do Replace $d_{l}(a)$ with

$\sum_{s \in S_{d}} i_{s}>0$ where $S_{d}$ is the set of local states of $m_{i}$ that satisfy $d_{l}(a)$

Replace $r_{l}(a)$ with

$\bigvee_{s \in S_{d}, t \in S_{r}, s \neq t}\left(i_{t}^{\prime}=i_{t}+1 \wedge i_{s}^{\prime}=i_{s}-1\right.$

$\left.\wedge \bigwedge_{p \neq s, p \neq t} i_{p}^{\prime}=i_{p}\right)$

$\vee \bigvee_{s \in\left(S_{d} \cap S_{r}\right)}\left(i_{s}^{\prime}=i_{s} \wedge \bigwedge_{p \neq s} i_{p}^{\prime}=i_{p}\right)$ where $S_{r}$ is the set of local states of $m_{i}$ that satisfy $r_{l}(a)$

Figure 4: Algorithm for counting abstraction

\subsection{Experimental Results}

Table 1 shows the performance of the Action Language Verifier for the Airport Ground Traffic Control monitor specification given in Figure 2. In the first column we denote the total number of processes in the specification. For example, the results from the first row are for the specification 
Table 1: Verification Results For Airport Ground Traffic Control Specification. xA (xD) denotes $x$ many arriving (departing) airplane processes and $\mathbf{x}=\mathbf{P}$ denotes arbitrary number of airplane processes. P1, P2, and $\mathrm{P3}$ are the properties given in Figure 2. CT and VT denote transition system construction time and property verification time (in seconds), respectively.

\begin{tabular}{|c|c|c|c|c|}
\hline Processes & CT & \multicolumn{3}{|c|}{ VT } \\
& & P1 & P2 & P3 \\
\hline \hline 2 & 0.81 & 0.42 & 0.28 & 0.69 \\
4 & 1.50 & 0.78 & 0.50 & 1.13 \\
8 & 3.03 & 1.53 & 0.99 & 2.22 \\
16 & 6.86 & 3.02 & 2.03 & 5.07 \\
2A,PD & 1.02 & 0.64 & 0.43 & 0.83 \\
4A,PD & 1.94 & 1.19 & 0.81 & 1.39 \\
8A,PD & 3.95 & 2.28 & 1.54 & 2.59 \\
16A,PD & 8.74 & 4.6 & 3.15 & 5.35 \\
PA,2D & 1.67 & 1.31 & 0.88 & 3.94 \\
PA,4D & 3.15 & 2.42 & 1.71 & 5.09 \\
PA,8D & 6.40 & 4.64 & 3.32 & 7.35 \\
PA,16D & 13.66 & 9.21 & 7.02 & 12.01 \\
PA,PD & 2.65 & 0.99 & 0.57 & 0.43 \\
\hline
\end{tabular}

in Figure 2 with 2 Airplane processes. CT denotes the time spent in constructing the composite symbolic representations for the transition relation and the initial states of the the input specification (including the parsing time). VT denotes the verification time for each property. Although the input is an infinite state system (since numC3, numRW16R, and numRW16L are unbounded variables) the verification time scales very well. This is due to the efficiency of the composite symbolic representation and the BDDs. If we had partitioned the transition system to eliminate the boolean variables (as is done in most infinite state model checkers) we would have obtained $2^{64}$ partition classes for the fourth instance in Table 1. Mapping the boolean variables to integers on the other hand would have created 64 more integer variables, increasing the cost of arithmetic constraint manipulation (which is not likely to scale as well as BDDs).

We used counting abstraction to verify the Airport Ground Traffic Control monitor specification for arbitrary number of arriving and departing airplanes. First we verified specifications for a fixed number of arriving airplanes and an arbitrary number of departing airplanes by using counting abstraction only on the states of departing airplanes. For example, the row 4A,PD in Table 1, denotes the case with 4 arriving airplanes and an arbitrary number of departing airplanes. Although counting abstraction generates an integer variable for each local state of an airplane process, the experimental results in Table 1 shows that it scales well. In fact, the case where both states of the arriving and the departing airplanes are abstracted (PA,PD) properties are verified faster compared to some other cases. This is possibly due to the fact that counting abstraction, in a way, simplifies the system by abstracting away the information about individual processes. For example, in the abstract transition system it is not possible to determine which airplane is in which state, we can only keep track of the number of airplanes in a particular state.

We verified a large number of concurrent system specifications using Action Language Verifier including other moni- tor specifications such as monitors for sleeping barber problem, readers-writers problem and bounded buffers. Our experimental results are reported in [20].

\section{SYNTHESIS OF MONITORS}

In the monitor specification given in Figure 2, the shared variables such as numRW16R and numC3 represent the resources that will be shared among multiple processes. Submodule Airplane specify the type of processes that will share these resources. Our goal is to generate a monitor class in Java from monitor specifications such as the one given in Figure 2. First, we will declare the shared variables of the monitor specification (for example, numRW16R and numC3 in Figure 2) as private fields of the monitor class. Hence, these variables will only be accessible to the methods of the monitor class.

We will not try to automatically generate code for the threads that will use the monitor. This would go against the modularization principle provided by the monitors. Rather, we will leave the assumption that the threads behave according to their specification as a proof obligation. In general, a submodule in a monitor specification (Figure 3) should specify the most general behavior of the corresponding thread, or, equivalently, it should specify the minimum requirements for the corresponding thread for the monitor to execute correctly. Since the specifications about the local behavior of the threads are generally straightforward (such as an Airplane process should not execute exitRW3 action before executing reqLand) we think that it would not be too difficult for the concurrent programmer to take the responsibility for meeting these specifications.

We will generate a monitor method corresponding to each action of each submodule in the monitor specification. Consider the action:

$$
\begin{aligned}
& \text { exitRW3: } p c=\text { touchDown and numC3 }=0 \text { and numC3 } 3^{\prime}=\text { numC3+1 } \\
& \text { and numRW16R'=numRW16R-1 and } p c^{\prime}=\operatorname{taxiTo16L3} ;
\end{aligned}
$$

We are not interested in the expressions on local variable pc of the submodule Airplane. As we discussed above, we are only generating code for the monitor class which only has access to the shared variables. For the action exitRW3 removing the expressions on the local variables leaves us with the expression

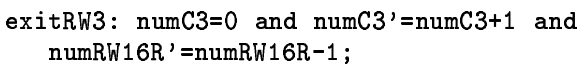

To implement this action as a monitor method we first have to check the guarding condition numC $3=0$ and then update numRW16R and numC3. However, if the guarding condition does not hold, we should wait until a process signals that the condition might have changed. A straightforward translation of this action to a monitor method would be

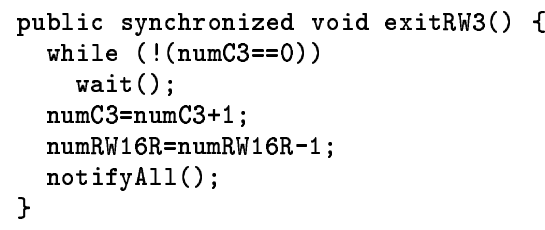

The reason we call the notifyAll method at the end is to wakeup processes that might be waiting on a condition related to variable numRW16R or numC3 which have just been 
updated by this action. Also note that the wait method is inside a while loop to make sure that the guard still holds after the thread wakes up. In the above translation, we used synchronized keyword to establish atomicity. Note that atomicity in Java is established only with respect to other methods or blocks which are also synchronized. So for this approach to work we have to make sure that shared variables are not modified by any part of the program which is not synchronized. We can establish this by declaring shared variables as private variables in the monitor class and making sure that all the methods of the monitor class are synchronized.

Using this straightforward approach, we can translate a monitor specification (based on the template given in Figure 3 ) to a Java monitor class using the following rules: 1) Create a monitor class with a private variable for each shared variable of the specification. 2) For each action in each submodule, create a synchronized method in the monitor class. 3 ) In the method for action $a$ start with a while loop which checks if $d_{s}(a)$ is true and waits if it is not. Then, put a set of assignments to update the variables according to the constraint in $r_{s}(a)$. After the assignments, call notifyAll method and exit. We will call this translation single-lock implementation of the monitor since it uses only this lock of the monitor class.

\subsection{Specific Notification Pattern}

The single-lock implementation described above is correct but it is inefficient [7, 18]. If we implement the Airport Ground Traffic Control monitor using the above scheme an exitRW3 action would awaken all the airplane threads that are sleeping. However, departing airplane threads should be awakened only when the number of airplanes on runway $16 \mathrm{~L}$ or one of the taxiways in $\mathrm{C} 3-\mathrm{C} 8$ changes (when one of the variables numRW16L, numC3, numC4, numC5, numC6, numC7, and numC8 become zero) and they do not need to be awakened on an update to status of runway 16R (when numRW16R is updated) or on entrance of an airplane into the taxiway C3 (when numC3 is incremented). Using different condition variables for each guarding condition improves the performance by awakening only related threads and eliminating the overhead incurred by context switch for threads which do not need to be awakened. In [7] using separate objects to wait and signal for separate conditions is described as a Java design pattern called specific notification pattern.

Figure 5 shows a fragment of the Java monitor that is automatically generated by our code generator from the Action Language specification of the Airport Ground Traffic Control monitor given in Figure 2 using specific notification pattern. The method for action exitRW3 calls Guard_exitRW3 method in a while loop till it returns true. If it returns false it waits on the condition variable exitRW3Cond. Any action that can change the guard for exitRW3 from false to true notifies the threads that are waiting on condition variable exitRW3Cond using exitRW3Cond. If the guard (numC3==0) is true then Guard_exitRW3 method decrements the number of airplanes using runway 16R (numRW16R=numRW16R-1) and increments the number of airplanes using taxiway C3 atomically and returns true. Since executing exitRW3 can only change action reqLand's guard from false to true, only threads that are waiting on condition variable reqLandCond are notified before method exitRW3 returns.

Action leave does not have a guard, i.e., its execution does not depend on the state of shared variables of the monitor. Hence, the method for action leave does not need to wait to decrement the number of airplanes on runway $16 \mathrm{~L}$ (numRW16L=numRW16L-1). After updating numRW16L however it notifies the threads waiting on the condition variables crossRW3Cond and reqTakeOffCond.

We will give an algorithm for generating Java code from monitor specifications in Action Language using specific notification pattern below. As stated before, we will assume that each action expression is in the form:

$$
\operatorname{EXP}(a) \equiv d_{l}(a) \wedge r_{l}(a) \wedge d_{s}(a) \wedge r_{s}(a)
$$

where $d_{l}(a)$ is an expression on unprimed local variables of module $m_{i}\left(\operatorname{VAR}\left(m_{i}\right)\right), r_{l}(a)$ is an expression on primed and unprimed local variables of $m_{i}, d_{s}(a)$ is an expression on unprimed shared variables $(\operatorname{VAR}(m))$, and $r_{s}(a)$ is an expression on primed and unprimed shared variables. Since we are not interested in the local states of the processes we will only use $d_{s}(a)$ and $r_{s}(a)$ in the code generation for the monitor methods. Let guard $_{a}$ denote a Java expression equivalent to $d_{s}(a)$. We will also assume that $r_{s}(a)$ can be written in the form $r_{s}(a) \equiv \bigwedge_{v \in \operatorname{RVAR}(a)} v^{\prime}=e_{v}$ where $e_{v}$ is an expression on domain variables in $\operatorname{VAR}(m)$. Let $a s s i g n_{a}$ denote a set of assignments in Java which correspond to $r_{s}(a)$.

To use the specific notification pattern in translating Action Language monitor specifications to Java monitors we need to associate the guard of each action with a lock specific to that action. Let $a$ be an action with a guard, guard and let cond $_{a}$ be the condition variable associated with $a$. The thread that calls the method that corresponds to action $a$ will wait on cond $_{a}$ when guard $_{a}$ evaluates to false. Any thread that calls a method that corresponds to another action, $b$, that can change the truth value of guard $_{a}$ from false to true will notify cond $d_{a}$. Hence, after an action execution, only the threads that are relevant to the updates performed by that action will be awakened.

The algorithm given in Figure 6 generates the information about the synchronization dependencies among different actions needed in the implementation of the specific notification pattern. For each action $a$ in each submodule $m_{i}$ the algorithm decides whether action $a$ is guarded or unguarded by checking the expression $d_{s}(a)$. If $d_{s}(a)$ is true (meaning that there is no guard) then the action is marked as unguarded. Otherwise, it is marked as guarded and a condition variable, cond $_{a}$, is created for action $a$. Execution of an unguarded action does not depend on the shared variables, hence, it does not need to wait on any condition variable. Next, the algorithm finds all the actions that should be notified after action $a$ is executed. We can determine this information by checking for each action $b \neq a$, if executing action $a$ when $d_{s}(b)$ is false can result in a state where $d_{s}(b)$ is true. If this is possible, then the condition variable created for action $b, \operatorname{cond}_{b}$, is added to the notification list of action $a$, which holds the condition variables that must be notified after action $a$ is executed.

Figure 7 shows translation of guarded and unguarded actions to Java [18]. For each guarded action $a$ a specific notification lock, cond $_{a}$ is declared and one private method and one public method is generated. The private method Guarded_Execute ${ }_{a}$ is synchronized on this object. If the guard of action $a$ is true then this method executes assignments in assign $_{a}$ and returns true. Otherwise, it returns false. Method Guarded_Wait ${ }_{a}$ first gets the lock for cond . $_{\text {. }}$ 


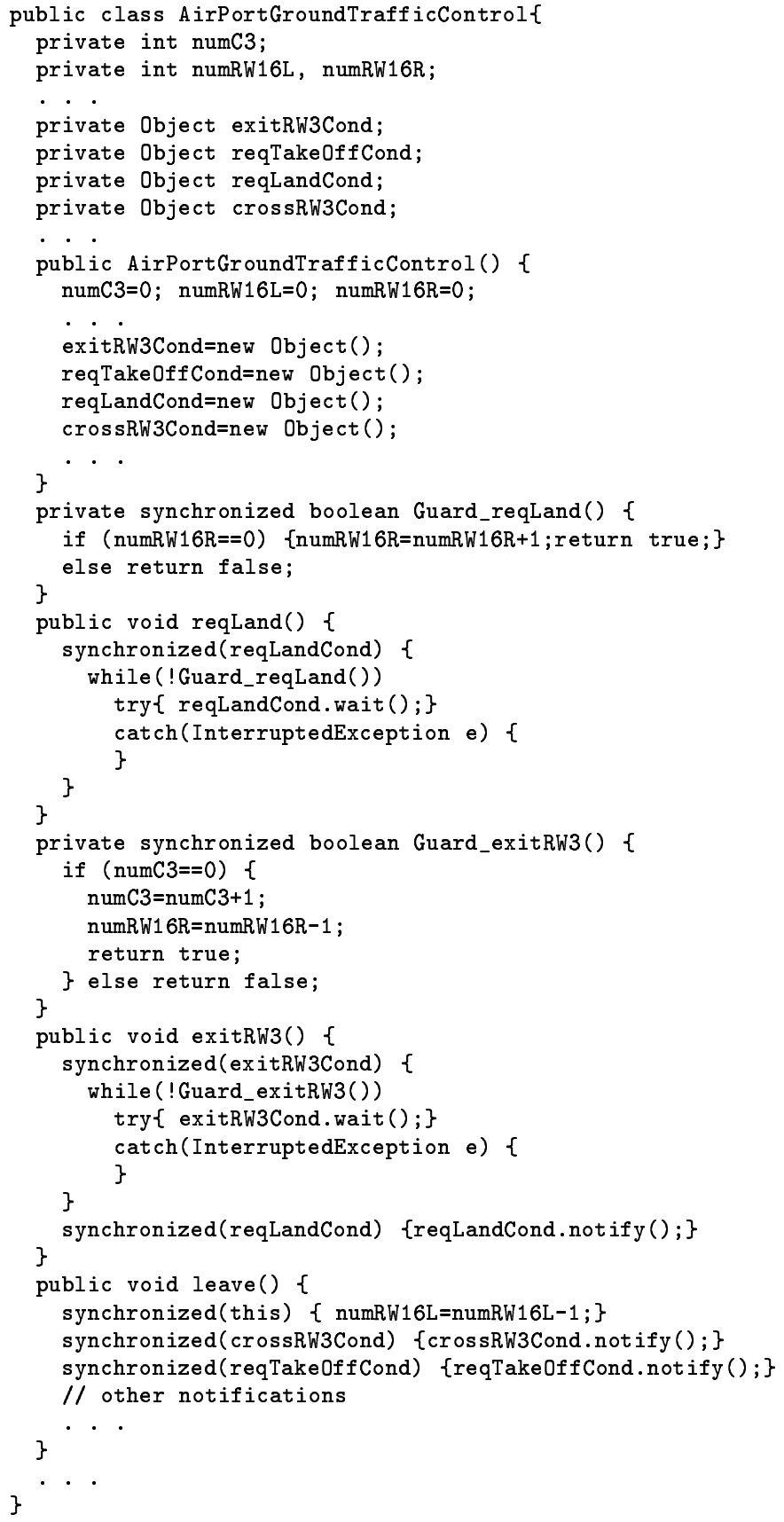

Figure 5: AirportGroundTrafficControl Class Using specific notification pattern

for each action $a$ do

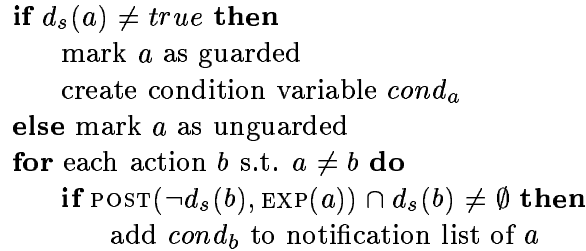

Figure 6: Extracting Synchronization Information

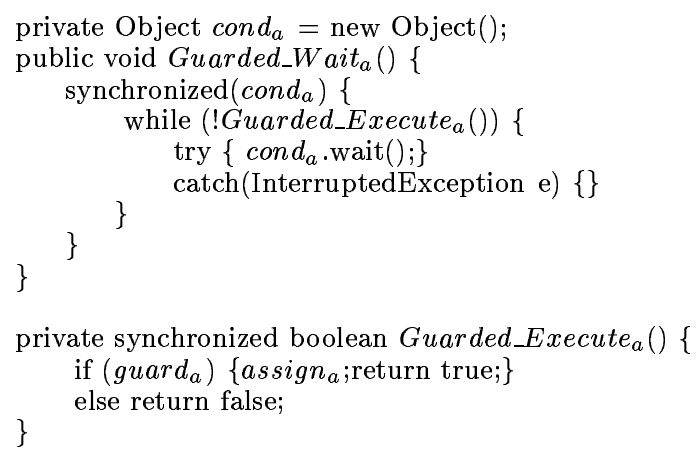

private synchronized boolean Guarded_Execute ${ }_{a}()\{$ if $\left(\right.$ guard $\left._{a}\right)\left\{\right.$ assign $_{a} ;$ return true; $\}$

(a)

public void Execute Ex $_{a}()$ synchronized(this) $\left\{\right.$ assign $\left.\left._{a} ;\right\}\right\}$

(b)

Figure 7: Translation of (a) guarded and (b) unguarded actions

Then it runs a while loop till Guarded_Execute ${ }_{a}$ method returns true. In the body of the while loop it waits on conda till it is notified by some thread that performs an update that can change truth value of method guard $_{a}$ and, there-

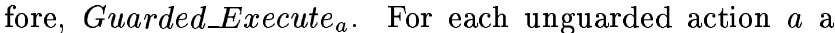
single public method Execute Ex $_{a}$ is produced. This method first acquires the lock for this object. Then executes the assignments assign $n_{a}$ of the corresponding action. Before exiting the public methods Guarded_Wait ${ }_{a}$ and Execute Ex $_{a}$ synchronized $\left(\operatorname{cond}_{b}\right)\left\{\operatorname{cond}_{b}\right.$.notifyAll(); $\}$ is executed for each action $b$ in the notification list of action $a$ (note that this is not shown in Figure 7).

The automatically generated Java monitor class should preserve the verified properties of the Action Language specification. This can be shown in two steps: 1) Showing that the verified properties are preserved by the single-lock implementation of the Action Language specification. 2) Showing the equivalence between the single-lock implementation and the specific notification pattern implementation. The proof of correctness of specific notification pattern (step 2) is given in [18]. The algorithm we give in Figure 6 extracts the necessary information in order to generate a Java monitor class that correctly implements the specific notification pattern.

Below, we will give a set of assumptions under which the monitor invariants that are verified on an Action Language specification of a monitor are preserved by its single-lock implementation as a Java monitor class.

1. Initial Condition: The set of program states immediately after the constructors of the monitor and the threads are executed satisfy the initial expression of the Action Language specification.

2. Atomicity: The observable states of the monitor are defined as the program states where this lock of the monitor is available, i.e., the states where no thread is active in the monitor.

3. Thread Behavior: The local behavior of the threads are correct with respect to the monitor specification. 
4. Scheduling: If there exists an enabled action then an enabled action will be executed.

Assuming that the above conditions hold we claim that the observable states of the single-lock implementation of the Action Language monitor specification satisfy the monitor invariants verified by the Action Language Verifier.

\section{CONCLUSIONS AND FUTURE WORK}

We think that our approach of combining specification, verification and synthesis, presented in this paper, can provide the right cost-benefit ratio for adaptation of automated verification techniques in practice. Writing a monitor specification has three major benefits: 1) It is a higher-level specification of a solution then a monitor implementation since it eliminates the need for condition variables and wait and signal operations. 2) Action Language specifications can be verified with Action Language Verifier. 3) Verified monitor specifications in Action Language can be automatically translated into Java monitor implementations where the correctness of the implementation is guaranteed by construction.

We are working on the integration of the automated counting abstraction algorithm to the Action Language Verifier. We think that our approach is applicable to interesting, real-world applications as demonstrated by our case study. For our approach to be applicable to a wider range of systems, we would like to extend our techniques to systems with boolean or integer arrays and recursive data structures (such as linked lists). We are working on both of these problems. We think that we can provide some analysis for arrays using uninterpreted functions. For analyzing specifications with recursive data structures, we are currently integrating the shape analysis technique [19] to Composite Symbolic Library. Our verification tools Composite Symbolic Library and Action Language Verifier are available at:

http://www.cs.ucsb.edu/ bultan/composite/

Acknowledgments: We would like to thank Aysu Betin for her help in the implementation of the automated code generation algorithm.

\section{REFERENCES}

[1] Statistical summary of commercial jet aircraft accidents, worldwide operations 1959-1995. Boeing Commercial Airplane Group, Airplane Safety Engineering, Seattle, Washington, 1996.

[2] R. Alur, T. A. Henzinger, and P. Ho. Automatic symbolic verification of embedded systems. IEEE Transactions on Software Engineering, 22(3):181-201, March 1996.

[3] G. R. Andrews. Concurrent programming, Principles and Practice. Benjamin/Cummings Publishing Co., 1991.

[4] T. Bultan. Action Language: A specification language for model checking reactive systems. In Proceedings of the 22nd International Conference on Software Engineering (ICSE 2000), pages 335-344, June 2000.

[5] T. Bultan, R. Gerber., and C. League. Composite model checking: Verification with type-specific symbolic representations. ACM Transactions of Software Engineering and Methodology, 9(1):3-50, January 2000.
[6] T. Bultan and T. Yavuz-Kahveci. Action Language Verifier. In Proceedings of the 16th IEEE International Conference on Automated Software Engineering, 2001.

[7] T. Cargill. Specific notification for Java thread synchronization. In International Conference on Pattern Languages of Programming, 1996.

[8] J. C.Corbett, M. B.Dwyer, J. Hatcliff, S. Laubach, C. S.Pasarenau, Robby, and H. Zheng. Bandera: Extracting finite-state models from Java source code. In Proc. 22nd Int. Conf. on Soft. Eng. (ICSE), 2000.

[9] G. Delzanno. Automatic verification of parameterized cache coherence protocols. In Proceedings of the 12th International Conference on Computer Aided Verification, volume 1855 of Lecture Notes in Computer Science, pages 53-68, 2000.

[10] G. Delzanno and T. Bultan. Constraint-based verification of client-server protocols. In Proceedings of the 7th International Conference on Principles and Practice of Constraint Programming, 2001.

[11] C. Demartini, R. Iosif, and R. Sisto. A deadlock detection tool for concurrent Java programs. Software-Practice and Experience, 29(7):577-603, 1999.

[12] X. Deng, M. B. Dwyer, J. Hatcliff, and M. Mizuno. Invariant-based specification, synthesis, and verification of synchronization in concurrent programs. In Proceedings of the 24th International Conference on Software Engineering (ICSE 2002), to appear.

[13] K. Havelund and T. Pressburger. Model checking Java programs using Java PathFinder. International Journal on Software Tools for Technology Transfer (STTT), 2000.

[14] C. Hoare. Monitors: An operating system structuring concept. Communications of the $A C M$, 17(10):549-557, 1974.

[15] T. Y. Kahveci, M. Tuncer, and T. Bultan. A library for composite symbolic representation. In Proceedings of the Seventh International Conference on Tools and Algorithms for the Construction and Analysis of Systems (TACAS 2001), 2001.

[16] D. Lea. Concurrent Programming in Java, Design Principles and Java. Sun Microsystems, 1999.

[17] K. L. McMillan. Symbolic model checking. Kluwer Academic Publishers, Massachusetts, 1993.

[18] M. Mizuno. A structured approach for developing concurrent programs in Java. Information Processing Letters, 1999.

[19] R. Wilhelm, M. Sagiv, and T. Reps. Shape analysis. In Proceedings of the 9th International Conference on Compiler Construction, volume 1781 of Lecture Notes in Computer Science, pages 1-17, 2000.

[20] T. Yavuz-Kahveci and T. Bultan. Heuristics for efficient manipulation of composite constraints. In A. Armando, editor, Proceedings of the 4 th International Workshop on Frontiers of Combining Systems (FroCos 2002), volume LNAI 2309, pages 57-71. Springer, 2002.

[21] C. Zhong. Modeling of Airport Operations Using An Object-Oriented Approach. $\mathrm{PhD}$ thesis, Virginia Polytechnic Institute and State University, 1997. 